\title{
LA.UR- $00-4667$
}

\section{Hadamard transform imaging spectrometer for pushbroom or staring use}

\author{
Ralph R. Berggren \\ Los Alamos National Laboretory, E526, Los Alamos, NM 87545 \\ Phone 505-667-0593, fax 505-665-6834, ralphb@lanigov
}

\begin{abstract}
A Hadamard transform spectrometer can be used in an imaging mode, meets most needs for hyperspectral imaging, provides the throughput advantage of an FTS, and has looser mechanical tolerances.
\end{abstract}

\section{Introduction}

A Hadamard transform spectrometer (HTS) is a dispersive spectrometer with the single slit replaced by a coded array of slits having transmissions of zero and one. After receiving a great deal of attention in the 1960's [1], interest in these devices faded, replaced by Fourier transform spectrometers that are not limited by the resolution of a grating. Both instruments have the advantage of providing high throughput. They receive radiation simultaneously from a large solid angle and a broad wavelength range.

A more recent HTS application [2] uses a 503-element diode array and a stationary slit array of 1005 elements to create a spectrometer with no moving parts. Another [3] uses a microslit array and a modulated two-dimensional detector array to create an imaging spectrometer.

Current requirements for remote sensing, on the order of $1.0 / \mathrm{cm}$ or better, can easily be obtained from a grating or prism. An investigation into the use of the Hadamard transform spectrometer with imaging detectors, and the creation of a simple computer model, suggested that the device would be nearly ideal for airborne or space applications. It would allow for use in either a staring or pushbroom mode and would avoid the need for the precision tolerances required by Fourier transform devices.

Equations are developed for the radiance on a detector element, summing all slits and all ground positions for pushbroom use or summing all slit positions for staring use.The method for determining the spectra for each ground pixel is developed, resulting in a Hadamard-like matrix and a simple linear solution.

Signal to noise ratio is difficult to quantify for any instrument. It depends on the details of the design and operation of an instrument, making comparisons complicated. However, the propagation of noise through the operation of an HTS and its data analysis have been modeled. As expected, the noise is reduced from that for a single slit by the square root of the number of slits.

\section{Principle of operation and calculation of radiance on each detector - pushbroom mode}

The figure below shows the operation of an HTS in a pushbroom mode. As an example, 7 detectors and 7 wavelengths are used. It is assumed that the dispersion is such that each separate wavelength from a given slit goes to a separate detector. The detectors are shown on the left. Let $\mathrm{d}$ enumerate the detectors, such that $-3<=\mathrm{d}<=3$.
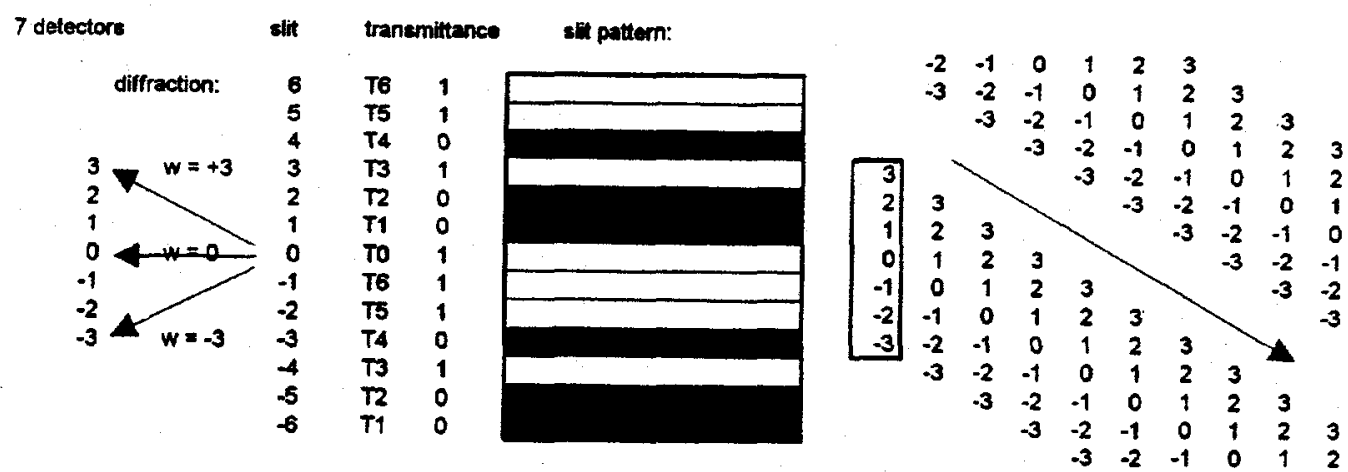


\section{DISCLAIMER}

This report was prepared as an account of work sponsored by an agency of the United States Government. Neither the United States Government nor any agency thereof, nor any of their employees, make any warranty, express or implied, or assumes any legal liability or responsibility for the accuracy, completeness, or usefulness of any information, apparatus, product, or process disclosed, or represents that its use would not infringe privately owned rights. Reference herein to any specific commercial product, process, or service by trade name, trademark, manufacturer, or otherwise does not necessarily constitute or imply its endorsement, recommendation, or favoring by the United States Government or any agency thereof. The views and opinions of authors expressed herein do not necessarily state or reflect those of the United States Government or any agency thereof. 


\section{DISCLAIMER}

Portions of this document may be illegible in electronic image products. Images are produced from the best available original document. 
Let $w$ enumerate the wavelengths, $-3<=w<=3$, with the wavelengths dispersed as shown by the arrows. In order to cover the 7 detectors there must be 13 slits, numbered $-6<s<=6$. The slits are assigned transmittances T0 to T6 as shown. As described below, a pattern of only 7 transmittances is needed. The transmittances and an illustration of the slit pattern are also shown.

Seven ground pixels, denoted by $\mathrm{g}$ where $-3<=\mathrm{g}<=3$, are shown. The pattern in the box denotes the positions relative to the slits at time $t=0$. The pattern is shown as the image moves across the slits at times denoted by $t$.

From this it can be seen that detector $d$ sees slit $s$ at wavelength $w$ where $s=d-w$. Also, slit $s$ sees ground pixel $\mathrm{g}$ where $\mathrm{g}=\mathrm{s}+\mathrm{t}$. Combining these, detector $\mathrm{d}$ sees ground pixel $\mathrm{g}=\mathrm{d}-\mathrm{w}+\mathrm{t}$ for wavelength $\mathrm{w}$ at time $\mathrm{t}$, with the transmittance of slit $d-w$.

Summing over all wavelengths from $w=-3$ to 3 gives the intensity at time $t$ on detector $d$ as:

$$
I(t, d)=\sum_{w=-3}^{3} T(d-w) H(d+t-w, w)
$$

The important next step is to set $\mathrm{d}+\mathrm{t}=\mathrm{n}$, a constant. Then the $\mathrm{H}$ function depends only on $\mathrm{n}$ and is the same for all values of $d$. Equation (1) can then be put in matrix form. Start by setting $t+d=0$. Then, using the values T0 to T6 given above as a function of detector position d gives:

$$
\left[\begin{array}{l}
I(-3,3) \\
I(-2,2) \\
I(-1,1) \\
I(0,0) \\
I(1,-1) \\
I(2,-2) \\
I(3,-3)
\end{array}\right]=\left|\begin{array}{ccccccc}
T 6 & T 5 & T 4 & T 3 & T 2 & T 1 & T 0 \\
T 5 & T 4 & T 3 & T 2 & T 1 & T 0 & T 6 \\
T 4 & T 3 & T 2 & T 1 & T 0 & T 6 & T 5 \\
T 3 & T 2 & T 1 & T 0 & T 6 & T 5 & T 4 \\
T 2 & T 1 & T 0 & T 6 & T 5 & T 4 & T 3 \\
T 1 & T 0 & T 6 & T 5 & T 4 & T 3 & T 2 \\
T 0 & T 6 & T 5 & T 4 & T 3 & T 2 & T 1
\end{array}\right| *\left|\begin{array}{c}
H(3,-3) \\
H(2,-2) \\
H(1,-1) \\
H(0,0) \\
H(-1,1) \\
H(-2,2) \\
H(-3,3)
\end{array}\right| \text { OCT } 262000
$$

By inverting the $T$-matrix the $H$-vector can be determined. Using different values for $t+d=n$ gives more equations for other sets of the H-vector, allowing all ground pixels at all wavelengths to be determined. This is equivalent to adding more columns to the I-matrix and the H-matrix.

\section{The Hadamard transform}

The T-matrix is cyclic and composed of only ones and zeros. It is fairly easy to create a cyclic matrix of that size that can be inverted, bit it will not generally be an optimum solution, since in general the matrix will not be wellconditioned. A Hadamard transform is a cyclic square matrix of ones and negative ones, having $2^{n}$ rows and columns, with the property that it is its own inverse [4]. These matrices cannot be used since slit transmissions of 1 are not possible. However, there are related square matrices of size $2^{n_{-1}}$ that have only ones and zeros. They are obtained from what are called "pseudo-random sequences [5]." There is a unique sequence for each of the $2^{n_{-}} 1$ sizes. For a length of 7 the sequence is $\{1,1,0,1,0,0,1\}$ or its reverse or any cyclic rotation. The $T$-matrix and its inverse are:

$$
\left|\begin{array}{lllllll}
1 & 1 & 0 & 1 & 0 & 0 & 1 \\
1 & 0 & 1 & 0 & 0 & 1 & 1 \\
0 & 1 & 0 & 0 & 1 & 1 & 1 \\
1 & 0 & 0 & 1 & 1 & 1 & 0 \\
0 & 0 & 1 & 1 & 1 & 0 & 1 \\
0 & 1 & 1 & 1 & 0 & 1 & 0 \\
1 & 1 & 1 & 0 & 1 & 0 & 0
\end{array}\right|=4 *\left|\begin{array}{ccccccc}
1 & 1 & -1 & 1 & -1 & -1 & 1 \\
1 & -1 & 1 & -1 & -1 & 1 & 1 \\
-1 & 1 & -1 & -1 & 1 & 1 & 1 \\
1 & -1 & -1 & 1 & 1 & 1 & -1 \\
-1 & -1 & 1 & 1 & 1 & -1 & 1 \\
-1 & 1 & 1 & 1 & -1 & 1 & -1 \\
1 & 1 & 1 & -1 & 1 & -1 & -1
\end{array}\right|
$$


The inverse has the zeros changed to negative ones, with the constant in front. The rows of the T-matrix share a property similar to orthonormality. The dot product of any row with itself is 4 , and with any other row is 2 . For the inverse, the dot product of any row with itself is 7 and with any other row is -1 . Solving for the H-vector requires multiplication by the inverse, and that involves only additions and subtractions.

\section{Error propagation and system tolerances}

A simple computer model was made in order to verify the solution and to study noise propagation. A spectrum was assumed, and a Gaussian random noise was added to the calculated detector response. The results showed, as expected since the equations are linear, that the standard deviation of the errors in the computed spectral values was the standard deviation of the noise reduced by the square root of the number of slits. As is usual with these multiplexed systems, detector noise is reduced. However, if the dominant noise is statistical photon noise and the spectrum is more or less uniform, the input noise increases with the square root of the number of slits and there is no gain.

\section{Operation in staring mode}

Referring to the figure, if the ground pixels are stationary relative to the instrument, then the slit must be moved. If the slit starts in the position shown at time $t=0$ and moves downward, then the detector $d$ sees slit $s$ at time $t$ and wavelength $w$ where $s=d-w+t$. The slit $s$ always sees ground pixel $g=s$. Therefore the detector $d$ sees ground pixel $g=s$ with the transmittance of slit $s=d-w+t$. Equation (1) gets changed to be:

$$
I(t, d)=\sum_{w=-3}^{3} T(d-w+t) H(d-w, w)
$$

For each staring measurement data is taken for 7 values of $t$ where $-3<=t<=3$. If we let either $d$ or $t$ be a constant, then the T-matrix is again cyclic, 7 values of $I$ are functions of 7 values of $H$, and by inverting the matrix the values of $\mathrm{H}$ can be determined.

\section{Conclusions}

In the pushbroom mode, there are no moving parts. Alignment tolerances for the slit array will be set relative to the size of the diffraction pattern at the image where the slits are located rather than by the wavelength of the radiation. Since the dispersive portion of a spectrometer usually operates at a higher f/number than the final focus, tolerances will be greatly increased. The same instrument can be used in either the pushbroom or staring mode with only the addition of a drive for the slit array. Using a transmissive slit array, tilt and axial tolerances will be relatively loose. Data processing requires only additions and subtractions, and the multiplexing advantages of a slitless spectrometer are obtained.

\section{References}

1. J. A. Decker, Jr., M. Harwit, "Experimental Operation of a Hadamard Spectrometer," Applied Optics 8, 2552-2554 (1969)

2. Joseph L. Robichaud, Wallace K. Wong, Roger A. Van Tassel, "Measured Performance of a Hadamard-coded Photodiode-array Spectrometer," in 8th International Conference on Fourier Transform Spectroscopy, Proc. SPIE 1575, 255-257 (1991)

3. Rainer Riesenberg and Ulich Dillner, "Hadamard imaging spectrometer with micro slit matrix" in Imaging Spectrometry V, Michael R. Descour and Sylvia S. Shen, eds, Proc. SPIE 3753, 203-213 (1999)

4. Richard Legault, "Reticles" in The Infrared \& Electro-Optical Systems Handbook, volume 3, Electro-Optical Components, William D. Rogatto, ed, Environmental Research Institute of Michigan, Ann Arbor, Michigan, and SPIE Optical Engineering Press, Belling ham, Washington, 1993) Chap. 9.

5. F. J. McWilliams and N. J. A. Sloane, "Pseudo-random sequences and arrays,", Proc. IEEE 64(12), 1715-1729 (1976) 